\title{
The Clinical Spectrum of Thyrotropin Receptor Gene (TSHR) Mutations
}

\author{
Yardena Tenenbaum-Rakover \\ $H a^{\prime}$ Emek Medical Center, Afula and The Ruth E Rappoprt \\ Faculty of Medicine, Technion, Haifa, \\ Israel
}

\section{Introduction}

Resistance to thyrotropin (RTSH) is a condition in which thyroid cells show reduced sensitivity to TSH. This condition is characterized by elevated serum TSH concentration, a normal or hypoplastic thyroid gland and normal to very low levels of thyroid hormones. Loss-of-function mutations in the TSH receptor gene (TSHR) lead to RTSH syndrome, presenting with either congenital hypothyroidism $(\mathrm{CH})$ or subclinical hypothyroidism (Beck-Peccoz et al., 2006; Refetoff, 2003).

$\mathrm{CH}$ occurs in about 1 in 3500 live births. Thyroid dysgenesis is responsible for $75 \%$ of these cases, dyshormonogenesis for $15 \%$, central hypothyroidism for $5 \%$, and $5 \%$ are due to other causes (Grüters et al., 2003; Kratzsch \& Pulzer, 2008). Most cases of $\mathrm{CH}$ due to thyroid dysgenesis occur sporadically, but $2 \%$ of the patients are familial (Castanet et al., 2000, 2001). Dyshormonogenesis is commonly recessively inherited (Park \& Chatterjee, 2005). Genes associated with thyroid gland dysgenesis include TITF1, TITF2 and PAX8 (De Felice \& Di Lauro, 2004; Gillam \& Kopp, 2001(a); Park \& Chatterjee, 2005). Thyroid dyshormonogenesis is caused by genes that are involved in thyroid hormone synthesis including thyroperoxidase (TPO), thyroglobulin (TG), sodium iodide symporter (NIS), pendrin (PDS), dual oxidase 2 (DUOX2) and its maturation factor (DUOXA2), and dehalogenase (DEHAL1) (Gillam \& Kopp, 2001(b); Grasberger \& Refetoff, 2010). Loss-of-function mutations in TSHR lead to a spectrum of phenotypes, depending on the mutation's location and severity (Biebermann et al., 2010; De Felice \& Di Lauro, 2004). The first report in 1968 of RTSH was of an 8-year-old boy with cretinism in whom the thyroid gland was small in a 99mTC scan and radioiodine uptake was normal (Stanbury et al., 1968). It was only in 1995 that the cause for RTSH syndrome in that case was shown to be a mutation in TSHR (Sunthornthepvarakul et al., 1995). Since the first report of $\mathrm{CH}$ caused by a TSHR mutation, several cases of loss-offunction mutations of TSHR have been reported: most are missense mutations, but deletions and insertions have been identified as well (see http: www.hgmd.cf.ac.uk/ac/ gene.php?gene=TSHR and OMIM\#275200) (Abramowicz et al., 1997; Alberti et al., 2002; Biebermann et al., 1997, 2010; Bretones et al., 2001; Camilot et al., 2005; Cangul et al., 2010; Clifton-Bligh et al., 1997; De Marco et al., 2009; de Roux et al., 1996; Fricke-Otto et al., 2005; Gagne et al., 1998; Grasberger et al., 2007; Jeziorowska et al., 2006; Jordan et al., 2003; Kanda et al., 2006; Nagashima et al., 2001; Narumi et al., 2009; Narumi et al., 2011; Park et al., 2004; 
Richter-Unruh et al., 2004; Rubio et al., 2008; Russo et al., 2000; Sriphrapradang et al., 2011; Sunthornthepvarakul et al., 1995; Sura-Trueba et al.,2009; Tenenbaum-Rakover et al., 2009; Tiosano et al., 1999; Tonacchera et al., 2000, 2001, 2004; Tsunekawa et al., 2006; Wonerow et al., 2001) (Table 1, Fig 1).

\section{TSHR: Structure and function}

TSH controls thyroid function upon its interaction with the G-protein-coupled TSHR. The family of G-protein-coupled receptors (GPCRs) shares seven transmembrane segments connected by three extracellular and three intracellular loops (ECL and ICL, respectively). Together with the receptors for glycoprotein hormones LH/HCG and FSH, TSHR has a long $\mathrm{N}$-terminal domain that is involved in recognition and binding of the ligand. The TSHR gene located on chromosome 14q31 was cloned in 1989 (Libert et al., 1989). It encodes a protein with a large N-terminal ligand-binding extracellular domain, a hepta-helical transmembrane domain and an intracellular domain. The extracellular domain is encoded by the first nine exons and part of exon 10, whereas the transmembrane and intracellular domains are encoded entirely by exon 10 . The protein consists of 744 amino acids and the Nterminal ectodomain consists of 398 amino acids composed of eight leucine-rich repeat motifs (Szkudlinski et al., 2002; Van Durme et al., 2006). Similar to other GPCRs, TSHR shares a common mode of intracellular signaling, stimulating the exchange of GDP for GTP on the Ga subunit (Gsa) and phosphoinositol (IP) turnover through Gq coupling. TSH binding to TSHR on the basolateral membrane of the thyroid follicular cells leads to stimulation of secondary-messenger pathways involving these two main pathways: Gs/cAMP, which mediates hormone secretion, thyroid cell growth and differentiation and iodide uptake, and IP/ $\mathrm{Ca}^{+2}$, which regulates thyroid hormone synthesis by stimulating iodide organification (Dumont et al., 1992; Vassart \& Dumont, 1992; Wonerow et al., 2001). Mutations in TSHR result in either gain or loss of receptor function.

\section{Gain-of-function mutations in TSHR}

Germline gain-of-function mutations result in non-autoimmune hyperthyroidism, whereas somatic mutations that constitutively activate TSHR result in toxic thyroid nodules. Hyperthyroidism caused by germline mutations in TSHR exhibits autosomal dominant inheritance. The mutations are located mainly in exon 10, which encodes the transmembrane region and intracellular tail that constitutively activate TSHR. The phenotype of these patients is characterized by hyperthyroidism with the presence of goiter but the absence of ophtolmopathy, and a lack of thyroid autoantibodies as well as of lymphocytic infiltration in thyroid histology (Van Sande et al., 1995). The clinical spectrum of phenotypes is variable and onset can occur anywhere from birth to adulthood. The presence of either congenital or adulthood-onset hyperthyroidism, multinodular goiter (MNG) and follicular carcinoma has been reported in the same family (Karges et al., 2005). To date, more then 55 germline gainof-function mutations have been reported, about 14 of them sporadic and the others with familial occurrence (http: www.hgmd.cf.ac.uk/ac/gene.php?gene=TSHR) (Akcurin et al., 2008; Davies et al., 2005; Farid et al., 2000; Führer et al., 1997(b); Holzapfel et al., 1997; Karges et al., 2005; Khoo et al., 1999; Tonacchera et al., 1996; Van Sande et al., 1995). Hyperthyroidism in affected individuals is often resistant to the conventional treatment used in Graves' disease, and either radiotherapy or total thyroidectomy is required. 
Autonomous benign and malignant toxic thyroid nodules have been shown to result from a variety of somatic mutations leading to constitutive activation of TSHR and affecting cell proliferation and cell function. Somatic TSHR mutations commonly occur in the transmembrane and ECL domains, but hot spots are the sixth transmembrane domain and the third ICL where the receptor interacts with G-proteins. Toxic adenoma due to TSHRactivating mutations may occur in infancy (Kohn et al., 2009) or even in utero (Kopp et al., 1997) (OMIM\#2603372) (Davies et al., 2005; Führer et al., 1997(a); Kohn et al., 2009). To date, about 25 different somatic TSHR-activating mutations have been reported manifesting with toxic adenoma, MNG and toxic thyroid carcinoma.

\section{Loss-of-function mutations in TSHR}

About 50 different loss-of-function mutations have been described in TSHR (Table 1). Affected individuals are either homozygous, compound heterozygous or heterozygous. The degree of insensitivity to TSH depends on the type and location of the TSHR mutation; more severe loss of TSHR function manifests as $\mathrm{CH}$, whereas mild mutations present with euthyroid hyperthyrotropinemia or subclinical hypothyroidism. When both alleles carry mutated receptors with complete lack of function, the result is severe hypothyroidism, commonly presenting at birth, whereas carriers of a mutation on one allele present with compensated hyperthyrotropinemia. The thyroid gland is hypoplastic or invisible in a 99mTC scan; however, in ultasonographic imaging, the gland is shown to be in a normal position and commonly of small size. TSHR mutations are distributed all along the receptor. Mutations located in the binding domain result in reduced binding capacity or decreased membrane expression of the receptor. The third ECL and the seventh intracellular domain of TSHR are hot spots for gain-of-function mutations, but some inactivating mutations have been identified in this domain as well (Alberti et al., 2002; Grasberger et al., 2007; Tiosano et al., 1999) (Fig. 1).

\subsection{Prevalence of loss-of-function mutations}

The exact prevalence of inactivating TSHR mutations is not known. A prevalence of $4.3 \%$ biallelic TSHR mutations was found among 134 Japanese infants with $\mathrm{CH}$ (Narumi et al., 2009). Among 38 children with non-autoimmune subclinical hypothyroidism, 11 (29\%) were carriers of TSHR mutations (Nicoletti et al., 2009). A prevalence of $12 \%$ TSHR mutations was shown in 42 subjects with non-autoimmune isolated hyperthyrotropinemia in Italy; all were with familial occurrence (Tonacchera et al., 2004). Camilot et al. (2005) identified 13 patients with heterozygous mutations $(11 \%)$ out of 116 pediatric patients with asymptomatic euthyroid hyperthyrotropinemia. A rate of $0.6 \%$ for carriers of W546X-mutated TSHR was identified in Welsh euthyroid individuals (Jordan et al., 2003). We found up to $2.4 \%$ carriers of two known mutations in a highly consanguineous population in the northern region of Israel (Tenebaum-Rakover et al., 2009). Moreover, the coexistence of two different novel mutations of TSHR in each of two separate clans has been shown (Sriphrapradang et al., 2011). In view of these data, it may be speculated that the occurrence of inactivating TSHR mutations in certain populations is not so rare, and therefore screening for TSHR mutations is indicated in cases with non-autoimmune subclinical hypothyroidism in those populations. 


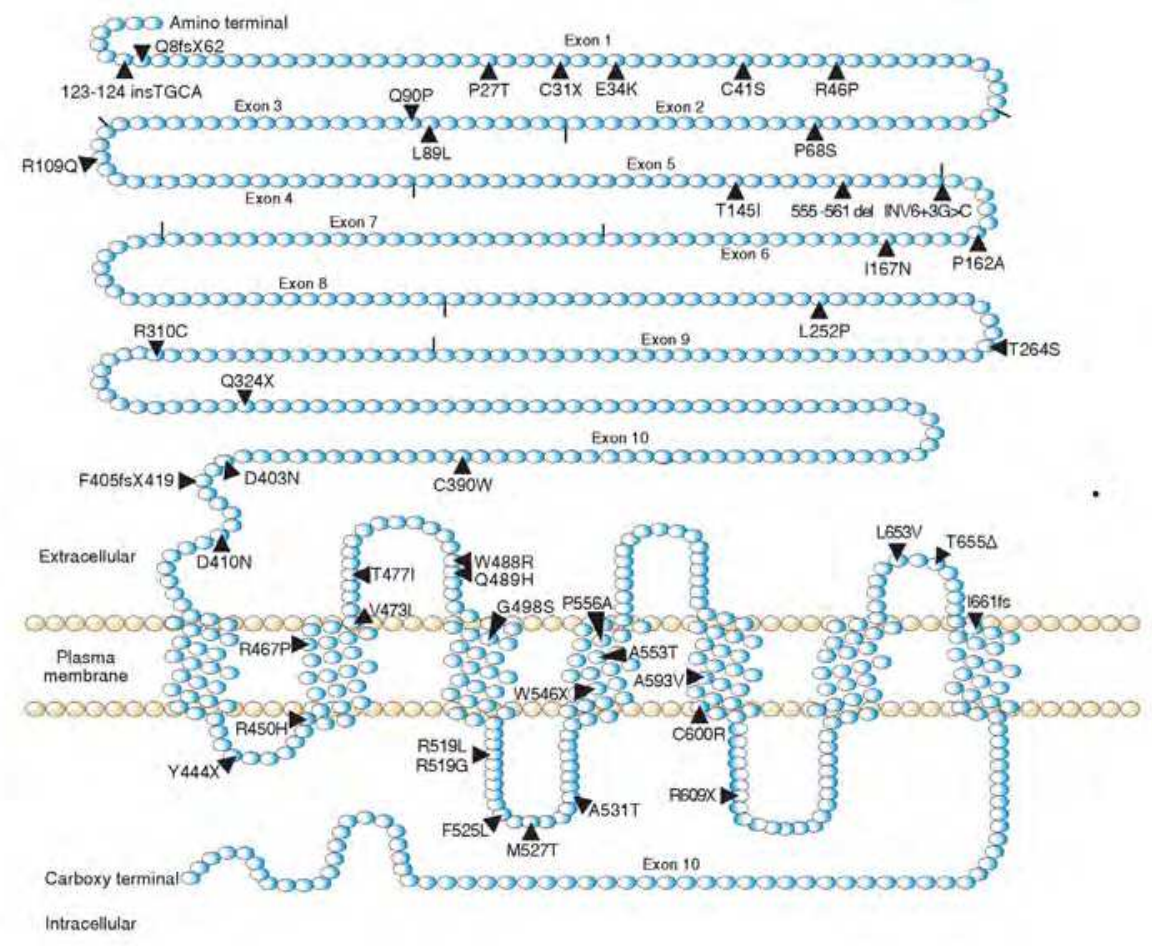

Fig. 1. Scheme of TSHR with known loss-of-function mutations

\subsection{Clinical characteristics}

Loss-of-function mutations manifest with a variable clinical spectrum of phenotypes. Severe uncompensated RTSH presents with $\mathrm{CH}$, partially compensated RTSH manifests with subclinical hypothyroidism, and fully compensated RTSH presents with euthyroid hyperthyrotropinemia or even normal thyroid function. The diagnosis of TSHR defect is based on the absence of thyroid antibodies, a lack of goiter, measurable serum thyroglobulin, and familial occurrence of hyperthyrotropinemia or hypothyroidism. $\mathrm{CH}$ is commonly detected by TSH-based neonatal screening but may missed by total $\mathrm{T}_{4}\left(\mathrm{TT}_{4)^{-}}\right.$ based screening since, in many cases, $\mathrm{TT}_{4}$ levels are within the normal range at birth (Table 1 ). The degree of $\mathrm{CH}$ is variable and depends on the genotype. Severe forms manifest as overt CH (Bretones et al., 2001; Gagne et al., 1998; Jeziorowska et al., 2006; Park et al., 2004; Tonacchera et al., 2000), moderate forms as hypothyroidism identified by neonatal screening without clinical symptoms of hypothyroidism (Abramowicz et al., 1997; Jordan et al., 2003), and mild forms present with hyperthyrotropinemia and normal thyroid hormones (de Roux et al., 1996; Nagashima et al., 2001; Narumi et al., 2009; Tenenbaum-Rakover et al., 2009). Gagne et al. (1998) described a case of $\mathrm{CH}$ with persistent neonatal jaundice, myxedematous facies, large fontanelle and absence of ossification centers of the knee on x-rays, indicating severe prenatal deficiency of thyroid hormone. Most of the described cases of $\mathrm{CH}$ are detected by neonatal screening with elevated $\mathrm{TSH}$ and normal $\mathrm{TT}_{4}$ levels, but without any 
clinical symptoms or signs of hypothyroidism (de Roux et al., 1996; Tenenbaum-Rakover et al., 2009). Nevertheless, $\mathrm{L}_{4} \mathrm{~T}_{4}$ therapy is initiated in most cases to prevent future consequences of untreated $\mathrm{CH}$. At the age of 2 to 3 years, when $\mathrm{L}^{-\mathrm{T}_{4}}$ is withdrawn, thyroid hormones remain low in the severe mutations (Abramowicz et al., 1997; Biebermann et al., 1997; Tonacchera et al., 2000); however in milder mutations, despite extremely elevated $\mathrm{TSH}$, thyroid hormone levels are normal, indicating compensated hypothyroidism (CliftonBligh et al., 1997; Tenenbaum-Rakover et al., 2009). 99mTC scan commonly reveals a normal or hypoplastic gland but in some cases, an absence of thyroid gland has been demonstrated, suggesting thyroid agenesis (Table 1). The presence of detectable thyroglobulin as well as the demonstration of a thyroid gland in the normal position in ultrasonographic imaging exclude thyroid agenesis and indicate a diagnosis of RTSH. In a few reports, an enlarged thyroid gland has been described (de Roux et al., 1996; Grasberger et al., 2007). Inactivating TSHR mutations at older ages present with either subclinical hypothyroidism or euthyroid hyperthyrotropinemia without thyroid autoantibodies. The affected patients are commonly identified by routine laboratory tests and are asymptomatic. Most of the described cases are heterozygous for TSHR mutations, but biallelic mutations have been reported as well (Kanda et al., 2006; Russo et al., 2000; Sriphrapradang et al., 2011; Tenenbaum-Rakover et al., 2009; Tonacchera et al., 2001, 2007).

\subsection{Mechanism of loss-of-function mutations}

The mechanism leading to loss-of-function of TSHR includes abnormal binding affinity, abnormal receptor synthesis, accelerated degradation, defective receptor targeting to the cell membrane and abnormal signal transduction (Tao, 2006). Mutations may exert their activity by causing protein misfolding, misassembly or aberrant oligomerization. Loss-of-function mutations are located all along the TSHR (Biebermann et al., 2010) (Fig 1). The function of TSHR is assessed in vitro by cAMP response, IP accumulation, TSH binding and cell-surface expression of the mutated receptor. The analysis is performed with COS-7 cells transfected with the mutant receptor. Each mutation has a different effect on binding capacity, membrane expression and cAMP and IP accumulation, depending on its type and location along the TSHR. In in-vitro studies, it has been shown that TSHR mutations differ in their effect on the Gs and Gq pathways, which may lead to more severe loss of one pathway compared to the other (Claus et al., 2005). The third ECL represents an important domain for intermolecular TSHR signal transduction and single amino acids play different roles in receptor folding and cAMP and IP signaling (Claus et al., 2005). We identified a biallelic L653V mutation located in the third ECL in three sisters presenting with marked hyperthyrotropinemia and increased thyroid radioiodine uptake (Grasberger et al., 2007). Normal ligand binding, slightly reduced cell expression and mildly reduced basal and stimulated cAMP accumulation with markedly reduced IP formation were found in in-vitro studies using transfected COS-7 cells. These in-vitro findings explained the phenotype of the affected subjects manifesting compensated hyperthyrotropinemia concomitant with increased iodide uptake, and this was the first report to provide in-vivo evidence of the important role of the IP/ $\mathrm{Ca}^{+2}$ pathway in the regulation of thyroid hormone synthesis. Narumi et al. (2011) recently reported two patients with $\mathrm{CH}$ and high iodide uptake harboring biallelic TSHR mutations (R450H+T145I in one and R450H+I166fs in the other), supporting our previous findings. They termed this apparently discrepant phenotype nonclassic TSH resistance. 


\begin{tabular}{|c|c|c|c|c|c|c|c|c|c|c|c|c|c|c|c|c|c|c|c|c|c|c|c|c|}
\hline 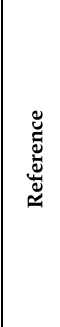 & 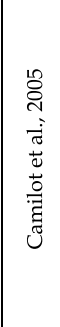 & 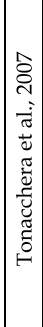 & 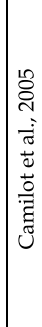 & 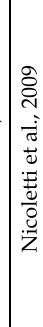 & 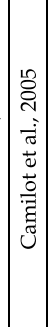 & 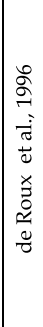 & 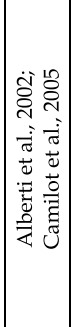 & 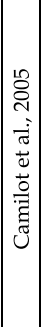 & 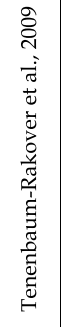 & 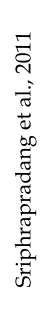 & 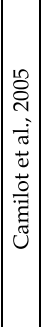 & 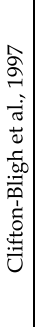 & 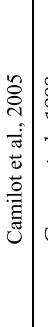 & 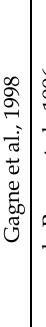 & 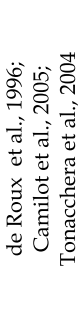 & 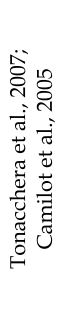 & 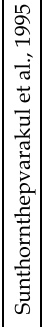 & 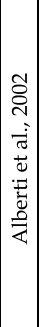 & 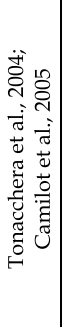 & 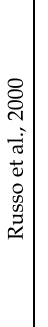 & 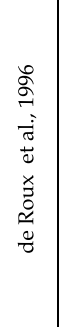 & 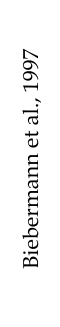 & 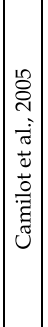 & 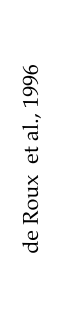 \\
\hline 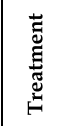 & $\mathbb{z}$ & 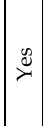 & 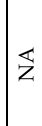 & $\mathscr{C}_{0}^{\infty}$ & $\overleftrightarrow{z}$ & $\stackrel{\circ}{z}$ & z & $\mathbb{z}$ & $\stackrel{2}{z}$ & 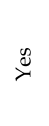 & $\overleftrightarrow{z}$ & $\stackrel{\substack{0 \\
\hdashline}}{ }$ & $\mathbb{z}$ & 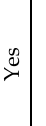 & 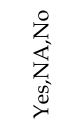 & $\stackrel{\mathscr{D}}{\underbrace{\prime}}$ & $\stackrel{\mathscr{C}}{0}$ & & $\overleftrightarrow{z}$ & 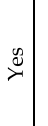 & $\stackrel{\infty}{\stackrel{\infty}{x}}$ & $\stackrel{\mathscr{D}}{\nearrow}$ & $\varangle$ & 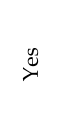 \\
\hline$\stackrel{*}{*}$ & $\mathbb{Z}$ & $\vec{c}$ & z & $\mathbb{z}$ & $\mathbb{z}$ & $\begin{array}{l}0 \\
\text { I }\end{array}$ & $\begin{array}{l}\vec{m} \\
\text { Dे }\end{array}$ & $\overleftrightarrow{z}$ & 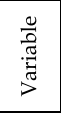 & 3 & $\mathbb{z}$ & $\begin{array}{l}3 \\
\infty\end{array}$ & $\mathbb{z}$ & $\begin{array}{l}\overrightarrow{0} \\
\stackrel{2}{r}\end{array}$ & 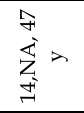 & $\vec{\infty}$ & $\begin{array}{l}\vec{z} \\
\stackrel{0}{\sim}\end{array}$ & in & ثे & $\overrightarrow{8}$ & $\begin{array}{l}\vec{M} \\
\stackrel{0}{*}\end{array}$ & 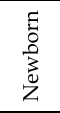 & $\overleftrightarrow{z}$ & $\begin{array}{l}\overline{0} \\
\text { in }\end{array}$ \\
\hline 㱐尊 & 艺 & 虾 & $\overleftarrow{z}$ & $\mathbb{z}$ & 要 & $\approx$ & $\stackrel{m}{\sigma}$ & $\mathbb{z}$ & $\begin{array}{l}\text { चี } \\
\text { Zू } \\
\text { Z }\end{array}$ & $\overleftrightarrow{z}$ & $\overleftrightarrow{z}$ & 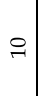 & 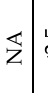 & $\stackrel{\substack{i \\
\mathrm{v}}}{\mathrm{v}}$ & 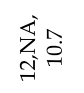 & $\begin{array}{l}\infty \\
\infty\end{array}$ & & 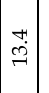 & $\widehat{్ త i ~}$ & $\stackrel{N}{\mathcal{I}}$ & సิ & $\stackrel{\infty}{\infty}$ & $\overleftrightarrow{z}$ & తి \\
\hline E & $\overleftrightarrow{\text { Z }}$ & $\overleftrightarrow{z}$ & $\overleftarrow{z}$ & & $\mathbb{z}$ & $\varangle$ & $\overleftarrow{z}$ & $\mathbb{z}$ & 艺 & $\stackrel{L}{=}$ & $\overleftrightarrow{z}$ & $\overleftrightarrow{z}$ & $\mathbb{z}$ & $\mathbb{z}$ & $\overleftrightarrow{z}$ & $\overleftrightarrow{z}$ & 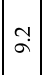 & $\mathbb{z}$ & $\overleftrightarrow{z}$ & $\overleftarrow{z}$ & 艺 & : & 乙 & 乙 \\
\hline 密疋 & $\mathbb{z}$ & 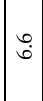 & $\overleftrightarrow{z}$ & $\overbrace{\infty}^{\infty}$ & $\mathbb{z}$ & సి & 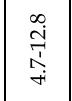 & $\overleftrightarrow{z}$ & 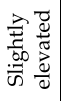 & $\mathbb{N}$ & $\overleftrightarrow{z}$ & $\sigma$ & $\begin{array}{l}n \\
0\end{array}$ & 品 & $\begin{array}{l}\overrightarrow{0} \\
\stackrel{2}{2} \\
\text { बे } \\
\text { مे }\end{array}$ & $\begin{array}{l}\infty \\
\infty \\
0 \\
0 \\
\infty \\
\infty\end{array}$ & f & 워 & $\stackrel{\infty}{\infty}^{\circ}$ & 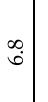 & $\mathbb{F}$ & $\infty$ & $\overleftrightarrow{z}$ & ल゙ \\
\hline 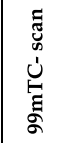 & $\mathbb{Z}$ & z & 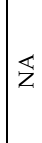 & $\mathbb{z}$ & $\overleftrightarrow{z}$ & 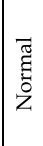 & 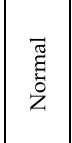 & $\mathbb{z}$ & $\begin{array}{l}\text { శే } \\
\text { हू } \\
\text { Z }\end{array}$ & 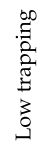 & $\overleftrightarrow{z}$ & 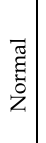 & $\overleftrightarrow{z}$ & 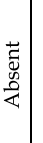 & $\begin{array}{l}\text { ]ే } \\
\text { हू } \\
\text { Z }\end{array}$ & $\overleftarrow{z}$ & $\mid \begin{array}{l}\vec{z} \\
\tilde{\Xi} \\
0 \\
Z \\
Z\end{array}$ & 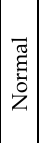 & $\overleftarrow{z}$ & 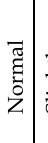 & 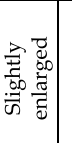 & $\overleftarrow{z}$ & $\mathbb{z}$ & 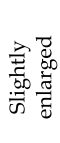 \\
\hline 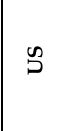 & $\mathbb{Z}$ & 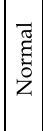 & ź & 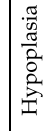 & 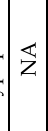 & $\overleftarrow{z}$ & $\begin{array}{l}\text { तే } \\
\text { हूँ } \\
\text { Zे }\end{array}$ & $\overleftrightarrow{Z}$ & $\begin{array}{l}\text { तే } \\
\text { हूँ } \\
\text { Z }\end{array}$ & $\begin{array}{l}\overline{\widetilde{\Xi}} \\
\text { हूँ } \\
\text { Zे }\end{array}$ & $\overleftrightarrow{z}$ & 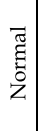 & $\overleftarrow{z}$ & $\begin{array}{l}\frac{\pi}{0} \\
\frac{\pi}{2} \\
\frac{2}{2} \\
\frac{2}{2} \\
\text { 空 }\end{array}$ & $\overleftarrow{z}$ & $\begin{array}{l}\text { ]ँ } \\
\text { हू } \\
\text { Z }\end{array}$ & $\overleftrightarrow{z}$ & 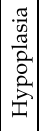 & $\begin{array}{l}\widetilde{\widetilde{\Xi}} \\
\text { है } \\
\text { Z }\end{array}$ & $\begin{array}{l}\overline{\widetilde{\Xi}} \\
\text { हूँ } \\
\text { Z }\end{array}$ & $\mathbb{z}$ & 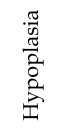 & $\overleftrightarrow{z}$ & $\mathbb{z}$ \\
\hline 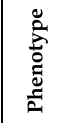 & 芯 & \begin{tabular}{|l}
$\mathbb{Z}$ \\
W
\end{tabular} & U. & $\underset{w}{I}$ & $\mathbb{J}$ & J & ت્己 & 己્ & ت्己 & $\vec{U}$ & 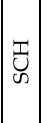 & $\mathbb{U}$ & J్ & త్ & 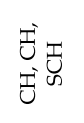 & 壳 & $\vec{U}$ & 胥 & $\underset{⿱ 乛 龰}{\mathbb{Z}}$ & U్ & J & త゙ & 胥 & $\underset{⿱ 乛 龰}{T}$ \\
\hline \multirow{2}{*}{ 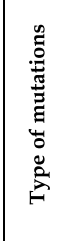 } & $\overline{3}$ & $\overrightarrow{3}$ & $\overrightarrow{3}$ & 3 & 3 & 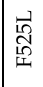 & $\vec{\xi}$ & $\overrightarrow{3}$ & $\vec{\xi}$ & 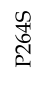 & $\overrightarrow{3}$ & $\begin{array}{l}x \\
0 \\
11 \\
3 \\
3\end{array}$ & $\overrightarrow{3}$ & 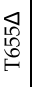 & $\begin{array}{l}\mathbb{\Xi} \\
\stackrel{\Xi}{\Omega}\end{array}$ & $\overrightarrow{3}$ & $\begin{array}{l}\mathrm{Z} \\
\mathrm{G} \\
=\end{array}$ & 总 & $\overrightarrow{3}$ & 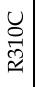 & 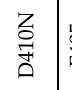 & 谣蓄 & $\overrightarrow{3}$ & 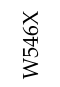 \\
\hline & 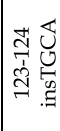 & 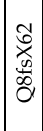 & 太心 & $\vec{\circlearrowright}$ & 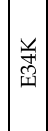 & F्ञ & $\stackrel{n}{\vec{J}}$ & $\begin{array}{l}\vec{t} \\
\vec{a} \\
\approx\end{array}$ & $\begin{array}{l}\text { 总 } \\
\text { : }\end{array}$ & 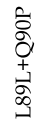 & $\mid \begin{array}{l}O \\
\circ \\
\stackrel{\approx}{\approx}\end{array}$ & $\begin{array}{l}9 \\
8 \\
\vec{a} \\
a\end{array}$ & 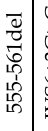 & 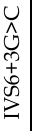 & $\begin{array}{l}\mathbb{\Xi} \\
\text { d } \\
\mathbb{E}\end{array}$ & $\begin{array}{l}\mathbb{d} \\
\stackrel{\Xi}{E}\end{array}$ & 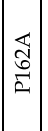 & \begin{tabular}{|l|}
$\mathbb{a}$ \\
$\mathbb{d}$ \\
$\mathbb{2}$ \\
\end{tabular} & 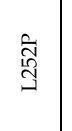 & $\begin{array}{l}U \\
\overrightarrow{0} \\
\approx \\
\approx\end{array}$ & 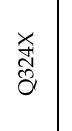 & $\begin{array}{l}3 \\
\text { 3. } \\
\text { రे }\end{array}$ & $\mid$\begin{tabular}{|l}
$Z$ \\
0 \\
0 \\
$o$ \\
0
\end{tabular} & $\begin{array}{l}3 \\
\text { 3. } \\
\text { రे }\end{array}$ \\
\hline
\end{tabular}




\begin{tabular}{|c|c|c|c|c|c|c|c|c|c|c|c|c|c|c|c|c|c|c|c|c|c|c|}
\hline 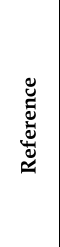 & 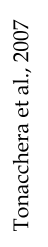 & 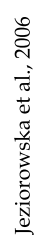 & 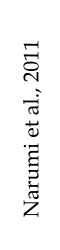 & 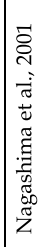 & 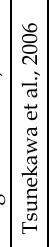 & 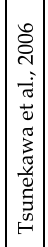 & 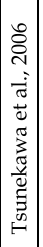 & 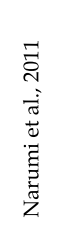 & 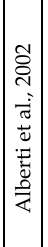 & 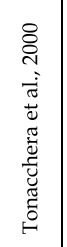 & 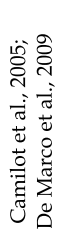 & 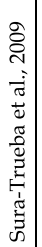 & 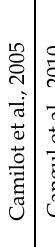 & 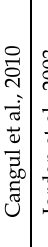 & 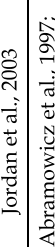 & 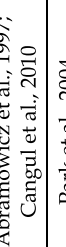 & 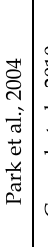 & & 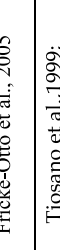 & 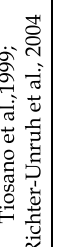 & 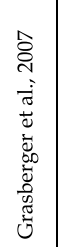 & 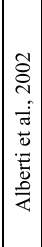 \\
\hline 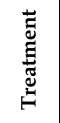 & $\stackrel{\mathscr{D}}{=}$ & $\overbrace{x}^{\mathscr{y}}$ & $\stackrel{\mathscr{X}}{x}$ & 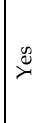 & $\mathbb{z}$ & $\overleftrightarrow{\mathrm{z}}$ & $\mathbb{z}$ & $\underset{ટ}{\mathscr{\lambda}}$ & $\mathbb{z}$ & $\overbrace{\nearrow}^{\infty}$ & $\overleftrightarrow{z}$ & 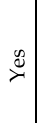 & $\overleftrightarrow{z}$ & $\overleftrightarrow{z}$ & 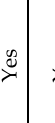 & 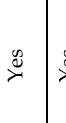 & 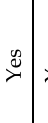 & 正 & 选 & 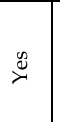 & $\stackrel{2}{z}$ & $\overleftrightarrow{z}$ \\
\hline$\stackrel{*}{*}$ & $\vec{m}$ & $\lambda$ & 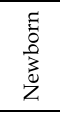 & İ & $\mathbb{z}$ & $\overleftrightarrow{z}$ & $\mathbb{z}$ & $\begin{array}{l}\text { E. } \\
\text { D. } \\
\text { 3. } \\
\text { ż }\end{array}$ & in & $\vec{ন}$ & $\overleftrightarrow{z}$ & 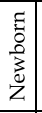 & 岁 & $\overleftrightarrow{z}$ & 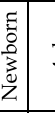 & $\vec{r}$ & 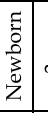 & & $\begin{array}{l}5 \\
0 \\
0 \\
3 \\
z \\
z \\
\end{array}$ & 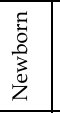 & $\vec{g}$ & $\overline{\tilde{n}}$ \\
\hline 框蔦 & $\approx$ & $\underset{\infty}{+1}$ & $\AA$ & $\begin{array}{l}\infty \\
\infty \\
\infty\end{array}$ & 望 & $\overleftrightarrow{z}$ & $\mathbb{z}$ & ले & $\nexists$ & 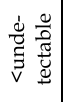 & $\overleftrightarrow{z}$ & - & $\mathbb{z}$ & $\begin{array}{l}\infty \\
\stackrel{\infty}{0} \\
\stackrel{0}{0}\end{array}$ & $\ltimes$ & 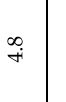 & & הֶ) & 志 & 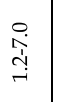 & 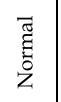 & I \\
\hline$\stackrel{\overline{0}}{\overline{0}}$ & $\overleftarrow{z}$ & $\overleftrightarrow{z}$ & $\overleftrightarrow{z}$ & $\overleftrightarrow{z}$ & $\mathbb{z}$ & $\begin{array}{l}\not \\
\infty \\
\infty\end{array}$ & $\begin{array}{l}a \\
\infty\end{array}$ & $\overleftarrow{z}$ & $\overleftrightarrow{z}$ & $\overleftarrow{z}$ & $\overleftarrow{z}$ & & $\mathbb{z}$ & $\mathbb{z}$ & 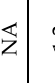 & $\stackrel{\sim}{\rightarrow}$ & $\underset{\sim}{-}$ & $\mathbb{z}$ & P. & $\overleftrightarrow{z}$ & $\overleftrightarrow{z}$ & $\overleftarrow{z}$ \\
\hline 密富 & $\stackrel{\infty}{\infty}$ & $\infty$ & חi & 罗 & 文 & $\hat{\vec{j}}$ & $\mid$\begin{tabular}{|c|}
$\vec{\infty}$ \\
$\infty$
\end{tabular} & $\stackrel{\infty}{ح}$ & $\stackrel{0}{\circ}$ & $\overleftrightarrow{z}$ & $\overleftrightarrow{z}$ & $\underset{\underset{\sim}{\infty}}{\stackrel{8}{2}}$ & 岁: & $m$ & 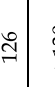 & ঙ্রি & $\stackrel{8}{\underset{C}{2}}$ & : & 6 & $\underset{\wedge}{8}$ & î & Iे \\
\hline 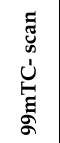 & $\overleftarrow{z}$ & 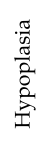 & 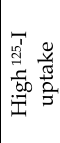 & 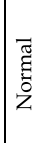 & 岁 & $\overleftrightarrow{z}$ & $\overleftrightarrow{z}$ & 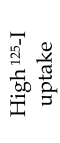 & 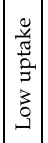 & 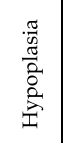 & $\overleftrightarrow{z}$ & 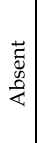 & $\overleftrightarrow{z}$ & $\overleftrightarrow{z}$ & 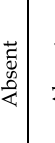 & $\begin{array}{l}\text { 苟 } \\
\text { d. }\end{array}$ & 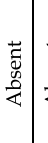 & 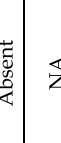 & $\mathbb{E}$ & $\begin{array}{l}\overrightarrow{\tilde{W}^{\prime}} \\
\text { 这 }\end{array}$ & 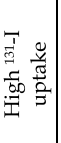 & 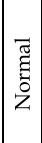 \\
\hline D & 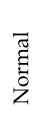 & 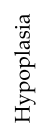 & $\overleftrightarrow{z}$ & 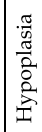 & 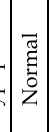 & $\overleftrightarrow{z}$ & $\mathbb{Z}$ & 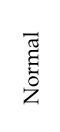 & $\begin{array}{l}\vec{z} \\
\tilde{\Xi} \\
\dot{0} \\
z\end{array}$ & 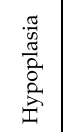 & 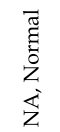 & 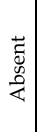 & 岁 & $\begin{array}{l}\overline{\widetilde{\Xi}} \\
\text { हू } \\
\text { z }\end{array}$ & $\begin{array}{c}\widetilde{\pi} \\
\tilde{z} \\
\tilde{z} \\
z\end{array}$ & 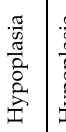 & 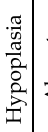 & & 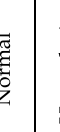 & 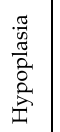 & 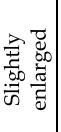 & $\begin{array}{l}\bar{\Xi} \\
\text { हू } \\
\check{Z}\end{array}$ \\
\hline 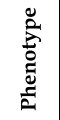 & 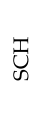 & Ü & J & J & 志 & 壳 & $\mid \begin{array}{l}\mathbb{Z} \\
\text { n }\end{array}$ & J & 胥 & J & J્ّ & J & 勇 & $\mathbb{J}$ & J゙ & I & च & 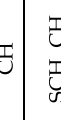 & E. & تే & $\begin{array}{l}\text { 志 } \\
\text { 出 } \\
\text { 志 }\end{array}$ & 壳 \\
\hline 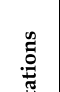 & $\overrightarrow{3}$ & 永 & 㞼 & $\begin{array}{l}\infty \\
\infty \\
0 \\
0 \\
0\end{array}$ & 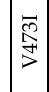 & 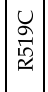 & $\mid \begin{array}{l}0 \\
\stackrel{2}{a} \\
\stackrel{2}{a}\end{array}$ & 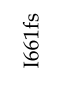 & $\overrightarrow{3}$ & 雬 & $\overrightarrow{3}$ & 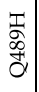 & $\overrightarrow{3}$ & 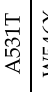 & 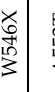 & 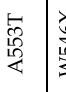 & $\begin{array}{l}x \\
6 \\
1 \\
\vdots \\
3 \\
3\end{array}$ & 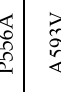 & $\begin{array}{l}\text { th } \\
\text { दे }\end{array}$ & 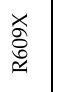 & 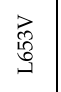 & $\overrightarrow{3}$ \\
\hline 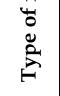 & 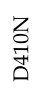 & 摩 & 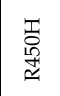 & \begin{tabular}{l}
$\frac{\pi}{0}$ \\
0 \\
0 \\
\hdashline
\end{tabular} & $\begin{array}{l}\mathbb{T} \\
\text { 㕝 } \\
\mathbb{2}\end{array}$ & 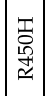 & 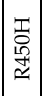 & 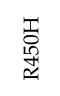 & 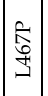 & 丐 & $\begin{array}{l}\text { 童 } \\
\text { o } \\
3 \\
3\end{array}$ & 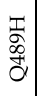 & 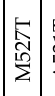 & 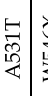 & 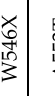 & 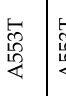 & 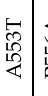 & 趈 & है & 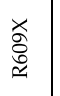 & $\begin{array}{l}\text { 希 } \\
\text { 忽 }\end{array}$ & 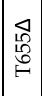 \\
\hline
\end{tabular}

* At diagnosis; NA, not available; $\mathrm{CH}$, congenital hypothyroidism, $\mathrm{SCH}$, sub-clinical hypothyroidism; $\mathrm{y}$, years; w, weeks; d, days; wt, wild type; US, ultrasonographic imaging

Table 1. Summary of the TSHR loss-of-function mutations 


\subsection{Heterozygosity for loss-of-function mutations}

Heterozygous subjects carrying loss-of-function mutations in TSHR are commonly mildly affected, presenting with euthyroid hyperthyrotropinemia but with variable expressivity (Camilot et al., 2005; Sriphrapradang et al., 2011; Tenenbaum-Rakover et al., 2009). Heterozygotes are typically diagnosed with slightly increased TSH but normal free $\mathrm{T}_{4}\left(\mathrm{FT}_{4}\right)$ levels. At least one case of neonatal hypothyroidism has been reported as well (Camilot et al., 2005). In in-vitro models expressing the combination of wild-type and mutated TSHR, it has been shown that basal and TSH-stimulated cAMP production are reduced compared to cells transfected with wild-type receptor, albeit less severely than in biallelic mutations. These in-vitro studies are consistent with the dominant-negative effect of the mutated receptor on the activity of the wild type and explain the mild phenotype of the carriers (Calebiro et al., 2005; Tenenbaum-Rakover et al., 2009). The dominant-negative effect of the mutated receptor may result in reduction of cAMP accumulation, as well as reduced membrane cell-surface expression of the receptor and retention in intracellular compartments (Calebiro et al., 2005).

\section{Genotype-phenotype association}

The phenotype of the affected subjects correlates with the severity of the mutation, which is dependent on its location and type, and whether it is mono- or biallelic. Most of the described cases reveal a direct association between the severity of the mutation and the phenotype, which is reflected by the extent of increase in TSH and decrease in $\mathrm{FT}_{4}$ levels. The more severe mutations manifest in infancy with persistent $\mathrm{CH}$, while the mild monoallelic mutations manifest as asymptomatic mild hyperthyrotropinemia. We identified 33 subjects carrying two novel TSHR gene mutations (P68S and L653V) in a large consanguineous kindred occurring as homozygous L653V (5 subjects), heterozygous P68S (4 subjects), heterozygous L653V (20 subjects), and compound heterozygous L653V/P68S (4 subjects). Our finding in a large cohort of affected members enabled us to assess the genotype-phenotype association. All homozygotes and compound heterozygotes presented with compensated RTSH, 9 out of 24 heterozygotes showed mild hyperthyrotropinemia and the others had normal TSH values. The clinical results were supported by in-vitro studies in which the L653V-mutated TSHR resulted in more severely impaired signal transduction than the other genotype combinations. However, large variability was found to exist between affected members. Among those with the homozygous L653V mutation, one child had $\mathrm{CH}$ and the other four, aged 3 to 20 years, had markedly elevated $\mathrm{TSH}$, but $\mathrm{FT}_{4}$ levels were within the normal range; among the heterozygous members for the two different mutations, variable hyperthyrotropinemia was observed, with a few of the affected subjects showing normal thyroid function (Tenenbaum-Rakover et al., 2009).

\section{Outcome}

Despite several reports of patients affected with TSHR mutations, there are limited data on the long-term outcome of this condition. In subjects with TSHR mutations, it has been shown that TSH levels remain stable and they do not develop hypothyroidism; in 
contrast, in autoimmune thyroid disease (AITD), overt hypothyroidism commonly develops over the years. In our abovementioned large cohort of affected family members, cross-sectional analysis showed neither a decrease nor an increase in TSH levels with age, suggesting stable compensated RTSH with an appropriately adjusted set point of pituitary-thyroid feedback (Tenenbaum-Rakover et al., 2009). In contrast to subclinical hypothyroidism in the context of AITD, the thyroidal compensation in mild to moderate RTSH is expected to be clinically stable with no progression toward true hypothyroidism or spontaneous regression toward normal TSH levels. Patients with homozygous or compound heterozygous mutations who are detected in infancy by neonatal screening to have $\mathrm{CH}$ may have normal $\mathrm{FT}_{4}$ levels despite elevated TSH levels after $\mathrm{L}_{-} \mathrm{T}_{4}$ withdrawal and in these patients, $\mathrm{L}-\mathrm{T}_{4}$ replacement may not be needed. In contrast, development of overt hypothyroidism at the age of 15 years was shown in a patient homozygous for the $\mathrm{R} 540 \mathrm{H}$ mutation who presented with compensated hypothyroidism in infancy (Mizuno et al., 2009), but not in an additional four subjects with the same genotype after long-term follow-up. Asymptomatic heterozygotes for TSHR mutations have normal or slightly elevated TSH levels with negative thyroid antibodies (Camilot et al., 2005). However, coexistence of thyroid autoantibodies has been reported in some cases of compensated RTSH, leading to overt hypothyroidism (Tonacchera et al., 2001). It is possible that carriers of TSHR mutations are at increased risk for AITD. TSHR is involved in AITD, TSH-stimulating autoantibodies in Graves' disease and TSH-blocking antibodies in Hashimoto thyroiditis. Therefore, it has been speculated that modification of TSH structure by the mutated receptor may lead to AITD (Tonacchera et al., 2001). Fluctuation of TSH levels from slightly above normal to normal values have been observed in some cases by us and others (Tenenbaum-Rakover et al., 2009; Tonacchera et al., 2001). In view of the variability in outcome among affected individuals, careful long-term follow-up is recommended.

\section{Treatment}

The question of whether to treat patients with TSHR mutations with $\mathrm{L}^{-\mathrm{T}_{4}}$ is a matter of debate (Utiger, 1995). In cases with loss-of-function mutations in TSHR presenting with $\mathrm{CH}$, early initiation of $\mathrm{L}^{-\mathrm{T}_{4}}$ therapy is recommended to prevent late-effect consequences of hypothyroidism as in other etiologies of $\mathrm{CH}$. However, withdrawal of $\mathrm{L}_{-} \mathrm{T}_{4}$ at the age of 2 to 3 years revealed transient hypothyroidism in some cases, putting the need for lifelong replacement therapy into question (Alberti et al., 2002; Tenenbaum-Rakover et al., 2009). Euthyroid hyperthyrotropinemia caused by TSHR mutations with mild to moderate loss of function maintains stable compensated RTSH and may not necessitate thyroid hormone replacement. Moreover, most patients with RTSH do not present with symptoms of hypothyroidism or with biochemical parameters of uncompensated hypothyroidism, such as elevated CPK and liver enzymes and hyperlipidemia (Tenenbaum-Rakover et al., 2009). The presence of normal $\mathrm{FT}_{4}$ levels argues against the need for replacement treatment, especially when inadvertent overtreatment, producing subclinical hyperthyroidism, can have undesirable effects (Samuels et al., 2008). In our long experience, no clinical benefit has been observed with $\mathrm{L}_{-} \mathrm{T}_{4}$ therapy. Contrasting with this approach, it has been shown that some subjects with RTSH have a slight decrease in $\mathrm{FT}_{4}$ levels compared to controls, although 
remaining within the normal range, which may point to subclinical hypothyroidism in these affected patients. In addition, the possibility of secondary pituitary enlargement in patients with extreme hyperthyrotropinemia may support $\mathrm{L}-\mathrm{T}_{4}$ replacement therapy. In view of the variability of phenotypes in different types of mutations, as well as between individuals with the same genotypes, it is recommended that careful follow-up and cautious administration of $\mathrm{L}^{-} \mathrm{T}_{4}$ be considered based on individual thyroid hormone levels in the clinical context.

\section{Differential diagnosis}

The diagnostic work-up of RTSH should exclude PAX8 mutations, which are characterized by thyroid dysgenesis associated with kidney abnormalities (Grüters et al., 2003; Park \& Chatterjee, 2005), and mutations in guanine nucleotide binding subunit 1 (GNAS1), which encodes Gsa subunit and causes pseudohypoparathyroidism (PHP) type Ia. The latter inactivating mutations in Gsa lead to a syndrome of resistance to multiple hormones, including TSH (Mantovani et al., 2002). Another form of RTSH is an autosomal dominantly inherited disease characterized by euthyroid hyperthyrotropinemia, for which the specific gene has not yet been identified. This condition has been linked to a locus on chromosome 15q25.3-26.1 (Grasberger et al., 2005(b)). In many of the cases with clinical characteristics of RTSH, no mutations have been found in TSHR, suggesting that additional genes are involved in RTSH syndrome (Xie et al., 1997). Bigenic defects in thyroid-synthesis pathways have been recently described. Coexistence of mutations in TPO (Sriphrapradang et al., 2011) and GNAS (Lado-Abeal et al., 2011), in addition to mutations in TSHR, has been reported in the same individuals. In those reports, the coexistence of mutated TPO and TSHR in the same individuals belonging to the same kindred did not aggravate the severity of the RTSH phenotype (Sriphrapradang et al., 2011); similar observations were made for the presence of a monoallelic TSHR mutation coexisting with a GNAS mutation (Lado-Abeal et al., 2011). It is therefore suggested that in cases where TSHR mutations do not explain the phenotype, additional genes that are involved in thyroid hormone synthesis be screened. RTSH must be differentiated from AITD (Ross, 2000), the most common cause of subclinical hypothyroidism in the adult population. The presence of autoantibodies as well as a typical hypoechogenic pattern of the thyroid in ultasonographic imaging support the diagnosis of AITD. This is important from a clinical standpoint since in RTSH, hyperthyrotropinemia is almost always stable while in AITD, hypothyroidism develops with time in about $30 \%$ of the cases.

\section{Conclusion}

To date, about 50 different TSHR mutations have been reported presenting with a spectrum of phenotypes ranging from overt $\mathrm{CH}$ to mild euthyroid hyperthyrotropinemia. Subjects with euthyroid hyperthyrotropinemia commonly have stable TSH levels and do not develop overt hypothyroidism with time. The phenotype correlates with the genotype as the latter is reflected by the severity of hyperthyrotropinemia and the decrease in $\mathrm{FT}_{4}$ levels. Screening for TSHR mutations should be considered in individuals with apparent non-autoimmune subclinical hypothyroidism. In view of the variability in phenotypes and in outcome among individuals in this condition, careful long-term follow-up is recommended and replacement 
therapy should be considered on an individual basis according to thyroid hormone levels in the clinical context.

\section{Acknowledgments}

Thanks to Camille Vainstein for professional language editing, to Dr. Zaki Kraiem for fruitful discussion and to Dr. Morad Khayat for useful assistance.

\section{References}

Abramowicz, M.J.; Duprez, L.; Parma, J.; Vassart, G. \& Heinrichs, C. (1997). Familial congenital hypothyroidism due to inactivating mutation of the thyrotropin receptor causing profound hypoplasia of the thyroid gland. The Journal of Clinical Investigation, Vol.99, No. 12, (June 1997), pp. 3018-3024, ISSN 0021-9738

Akcurin, S.; Turkkahraman, D.; Tysoe, C.; Ellard, S.; De Leener, A.; Vassart, G. \& Costagliola, S. (2008). A family with a novel TSH receptor activating germline mutation (p.Ala485Val). European Journal of Pediatrics, Vol.167, No.11, (November 2008), pp. 1231-1237, ISSN 0340-6199

Alberti, L.; Proverbio, M.C.; Costagliola, S.; Romoli, R.; Boldrighini, B.; Vigone, M.C.; Weber, G.; Chiumello, G.; Beck-Peccoz, P. \& Persani, L. (2002). Germline mutations of TSH receptor gene as cause of nonautoimmune subclinical hypothyroidism. Journal of Clinical Endocrinology and Metabolism, Vol.87, No.6, (June 2002), pp. 2549-2555, ISSN 0021-972X

Beck-Peccoz, P.; Persani, L.; Calebiro, D.; Bonomi, M.; Mannavola, D. \& Campi, I. (2006). Syndromes of hormone resistance in the hypothalamic-pituitary-thyroid axis. Best Practice \& Research: Clinical Endocrinology \& Metabolism, Vol.20, No.4, (December 2006), pp. 529-546, ISSN 1521-690X

Biebermann, H.; Schöneberg, T.; Krude, H.; Schultz, G.; Gudermann, T. \& Grüters, A. (1997). Mutations of the human thyrotropin receptor gene causing thyroid hypoplasia and persistant congenital hypothyroidism. Journal of Clinical Endocrinology and Metabolism, Vol.82, No.10, (October 1997), pp. 3471-3480, ISSN 0021-972X

Biebermann, H.; Winkler, F. \& Kleinau, G. (2010). Genetic defects, thyroid growth and malfunctions of the TSHR in pediatric patients. Frontiers in Bioscience, Vol.15, No.3, (June 2010), pp. 913-933, ISSN 1093-9946

Bretones, P.; Duprez, L.; Parma, J.; David, M.; Vassart, G. \& Rodien, P. (2001). A familial case of congenital hypothyroidism caused by a homozygous mutation of the thyrotropin receptor gene. Thyroid, Vol.11, No.10, (October 2001), pp. 977-980, ISSN 1557-9077

Calebiro, D.; de Filippis, T.; Lucchi, S.; Covino, C.; Panigone, S.; Beck-Peccoz, P.; Dunlap, D. \& Persani, L. (2005). Intracellular entrapment of wild-type TSH receptor by oligomerization with mutants linked to dominant TSH resistance. Human Molecular Genetics, Vol.14, No.20, (October 2005), pp. 2991-3002, ISSN 0964-6906

Camilot, M.; Teofoli, F.; Gandini, A.; Franceschi, R.; Rapa, A.; Corrias, A.; Bona, G.; Radetti, G. \& Tato, L. (2005). Thyrotropin receptor gene mutations and TSH resistance: 
variable expressivity in the heterozygotes. Clinical Endocrinology, Vol.63, No.2, (August 2005), pp. 146-151, ISSN 1365-2265

Cangul, H.; Morgan, N.V.; Forman, J.R.; Saglam, H.; Aycan, Z.; Yakut, T.; Gulten, T.; Tarim, O.; Bober, E.; Cesur, Y.; Kirby, G.A.; Pasha, S.; Karkucak, M.; Eren, E.; Cetinkaya, S.; Bas, V.; Demir, K.; Yuca, S.A.; Meyer, E.; Kendall, M.; Hogler, W.; Barrett, T.G. \& Maher, E.R. (2010). Novel TSHR mutations in consanguineous families with congenital nongoitrous hypothyroidism. Clinical Endocrinology, Vol.73, No.5, (November 2010), pp. 671-677, ISSN 1365-2265

Castanet, M.; Lyonnet, S.; Bonaiti-Pellie, C.; Polak, M.; Czernichow, P. \& Leger J. (2000). Familial forms of thyroid dysgenesis among infants with congenital hypothyroidism. New England Journal of Medicine, Vol.343, No.6, (August 2000), pp. 441-442, ISSN 0028-4793

Castanet, M.; Polak, M.; Bonaiti-Pellie, C.; Lyonnet, S.; Czernichow, P. \& Leger, J. (2001). Nineteen years of national screening for congenital hypothyroidism: familial cases with thyroid dysgenesis suggest the involvement of genetic factors. Journal of Clinical Endocrinology and Metabolism, Vol.86, No.5, (May 2001), pp. 2009-2014, ISSN 0021-972X

Claus, M.; Jaeschke, H.; Kleinau, G.; Neumann, S.; Krause, G. \& Paschke, R. (2005). A hydrophobic cluster in the center of the third extracellular loop is important for thyrotropin receptor signaling. Endocrinology, Vol.146, No.12, (December 2005), pp. 5197-5203, ISSN 0013-7227

Clifton-Bligh, R.J.; Gregory, J.W.; Ludgate, M.; John, R.; Persani, L.; Asteria, C.; Beck-Peccoz, P. \& Chatterjee, V.K.K. (1997). Two novel mutations in the thyrotropin (TSH) receptor gene in a child with resistance to TSH. Journal of Clinical Endocrinology and Metabolism, Vol.82, No.4, (April 1997), pp. 1094-1100, ISSN 0021-972X

Davies, T.F.; Ando, T.; Lin, R.Y.; Tomer, Y. \& Latif, R.J. (2005). Thyrotropin receptorassociated diseases: from adenomata to Graves disease. The Journal of Clinical Investigation, Vol.115, No.8, (August 2005), pp. 1972-1983, ISSN 0021-9738

De Felice, M. \& Di Lauro, R. (2004). Thyroid development and its disorders: genetics and molecular mechanisms. Endocrine Reviews, Vol.25, No.5, (October 2004), pp. 722746, ISSN 0163-769X

De Marco, G.; Agretti, P.; Camilot, M.; Teofoli, F.; Tatò, L.; Vitti, P.; Pinchera, A. \& Tonacchera, M. (2009). Functional studies of new TSH receptor (TSHr) mutations identified in patients affected by hypothyroidism or isolated hyperthyrotrophinaemia. Clinical Endocrinology, Vol.70, No.2, (February 2009), pp. 335-338, ISSN 1365-2265

de Roux, N.; Misrahi, M.; Brouner, R.; Houang, M.; Carel, J.C.; Granier, M.; Le Bouc, Y.; Ghinea, N.; Boumedienne, A.; Toublanc, J.E. \& Milgrom, E. (1996). Four families with loss of function mutations of the thyrotropin receptor. Journal of Clinical Endocrinology and Metabolism, Vol.81, No.12 (December 1996), pp. 4229-4235, ISSN 0021-972X

Dumont, J.E.; Lamy, F.; Roger, P. \& Maenhaut, C. (1992). Physiological and pathological regulation of thyroid cell proliferation and differentiation by thyrotropin and other factors. Physiological Reviews, Vol.72, No.3, (July 1992), pp. 667-697, ISSN 0031-9333 
Farid, N.R.; Kascur, V. \& Balazs, C. (2000). The human thyrotropin receptor is highly mutable: a review of gain-of-function mutations. European Journal of Endocrinology, Vol.143, No.1, (July 2000), pp. 25-30, ISSN 0804-4643

Fricke-Otto, S.; Pfarr, N.; Mühlenberg, R. \& Pohlenz, J. (2005). Mild congenital primary hypothyroidism in a Turkish family caused by a homozygous missense thyrotropin receptor (TSHR) gene mutation (A593 V). Experimental and Clinical Endocrinology $\mathcal{E}$ Diabetes, Vol.113, No.10, (December 2005), pp. 582-585, ISSN 0947-7349

Führer, D.; Holzapfel, H.P.; Wonerow, P.; Scherbaum, W.A. \& Paschke, R. (1997a). Somatic mutations in the thyrotropin receptor gene and not in the Gs alpha protein gene in 31 toxic thyroid nodules. Journal of Clinical Endocrinology and Metabolism, Vol.82, No.11, (November 1997), pp. 3885-3891, ISSN 0021-972X

Führer, D.; Wonerow, P.; Willgerodt, H. \& Paschke, R. (1997b). Identification of a new thyrotropin receptor germline mutation (Leu629Phe) in a family with neonatal onset of autosomal dominant nonautoimmune hyperthyroidism. Journal of Clinical Endocrinology and Metabolism, Vol.82, No.12, (December 1997), pp. 4234-4238, ISSN 0021-972X

Gagne, N.; Parma, J.; Deal, C.; Vassart, G. \& van Vliet, G. (1998). Apparent congenital athyreosis contrasting with normal plasma thyroglobulin levels and associated with inactivating mutations in the thyrotropin receptor gene: are athyreosis and ectopic thyroid distinct entities? Journal of Clinical Endocrinology and Metabolism, Vol.83, No.5, (May 1998), pp. 1771-1775, ISSN 0021-972X

Gillam, M.P. \& Kopp, P. (2001a). Genetic regulation of thyroid development. Current Opinion in Pediatrics, Vol.13, No.4, (August 2001), pp. 358-363, ISSN 1040-8703

Gillam, M.P. \& Kopp, P. (2001b). Genetic defects in thyroid hormone synthesis. Current Opinion in Pediatrics, Vol.13, No.4, (August 2001), pp. 364-372, ISSN 1040-8703

Grasberger, H. \& Refetoff, S. (2010). Congenital defects of thyroid hormone synthesis. In: Genetic Diagnosis of Endocrine Disorders, R.E. Weiss, S. Refetoff, (Eds.), 1st ed., 87-95, Academic Press, ISBN 012-374-430X, Burlington, Massachusetts, USA

Grasberger, H.; Mimouni-Bloch, A.; Vantyghem, M.C.; van Vliet, G.; Abramowicz, M.; Metzger, D.L.; Abdullatif, H.; Rydlewski, C.; Macchia, P.E.; Scherberg, N.H.; van Sande, J.; Mimouni, M.; Weiss, R.E.; Vassart, G. \& Refetoff, S. (2005a). Autosomal dominant resistance to thyrotropin as a distinct entity in five multigenerational kindreds: clinical characterization and exclusion of candidate loci. Journal of Clinical Endocrinology and Metabolism, Vol.90, No.7, (July 2005), pp. 4025-4034, ISSN 0021972X

Grasberger, H.; Vaxillaire, M.; Pannain, S.; Beck, J.C.; Mimouni-Bloch, A.; Vatin, V.; Vassart, G.; Froguel, P. \& Refetoff, S. (2005b). Identification of a locus for nongoitrous congenital hypothyroidism on chromosome 15q25.3-26.1. Human Genetics, Vol.118, No.3-4, (December 2005), pp. 348-355, ISSN 0340-6717

Grasberger, H.; Van Sande, J.; Hag-Dahood Mahameed, A.; Tenenbaum-Rakover, Y. \& Refetoff, S. (2007). A familial TSH receptor mutation provides in vivo evidence that the inositol phosphates/ $\mathrm{Ca}^{2+}$ cascade mediates TSH action on thyroid hormone synthesis. Journal of Clinical Endocrinology and Metabolism, Vol.92, No.7, (July 2007), pp. 2816-2820, ISSN 0021-972X 
Grüters, A.; Biebermann, H. \& Krude, H. (2003). Neonatal thyroid disorders. Hormone Research, Vol.59, Suppl.1, (January 2003), pp. 24-29, ISSN 0301-0163

Holzapfel, H.P.; Wonerow, P.; von Petrykowski, W.; Henschen, M.; Scherbaum, W.A. \& Paschke, R. (1997). Sporadic congenital hyperthyroidism due to a spontaneous germline mutation in the thyrotropin receptor gene. Journal of Clinical Endocrinology and Metabolism, Vol.82, No.11, (November 1997), pp. 3879-3884, ISSN 0021-972X

Jeziorowska, A.; Pniewska-Siark, B.; Brzeziańska, E.; Pastuszak-Lewandoska, D. \& Lewiński, A. (2006). A novel mutation in the thyrotropin (thyroid-stimulating hormone) receptor gene in a case of congenital hypothyroidism. Thyroid, Vol.16, No.12, (December 2006), pp. 1303-1309, ISSN 1557-9077

Jordan, N.; Williams, N.; Gregory, J.W.; Evans, C.; Owen, M. \& Ludgate, M. (2003). The W546X mutation of the thyrotropin receptor gene: potential major contributor to thyroid dysfunction in a Caucasian population. Journal of Clinical Endocrinology and Metabolism, Vol.88, No.3, (March 2003), pp. 1002-1005, ISSN 0021-972X

Kanda, K.; Mizuno, H.; Sugiyama, Y.; Imamine, H.; Togari, H. \& Onigata, K. (2006). Clinical significance of heterozygous carriers associated with compensated hypothyroidism in $\mathrm{R} 450 \mathrm{H}$.; a common inactivating mutation of the thyrotropin receptor gene in Japanese. Endocrine, Vol.30, No.3, (December 2006), pp. 383-388, ISSN 0969-711X

Karges, B.; Krause, G.; Homoki, J.; Debatin, K.M.; de Roux, N. \& Karges, W. (2005). TSH receptor mutation V509A causes familial hyperthyroidism by release of interhelical constraints between transmembrane helices TMH3 and TMH5. Journal of Endocrinology, Vol.186, No.2, (August 2005), pp. 377-385, ISSN 0022-0795

Khoo, D.H.; Parma, J.; Rajasoorya, C.; Ho, S.C. \& Vassart, G. (1999). A germline mutation of the thyrotropin receptor gene associated with thyrotoxicosis and mitral valve prolapse in a Chinese family. Journal of Clinical Endocrinology and Metabolism, Vol.84, No.4, (April 1999), pp. 1459-1462, ISSN 0021-972X

Kohn, B.; Grasberger, H.; Lam, L.L.; Ferrara, A.M. \& Refetoff, S. (2009). A somatic gain-offunction mutation in the thyrotropin receptor gene producing a toxic adenoma in an infant. Thyroid, Vol.19, No.2, (February, 2009), pp. 187-191, ISSN 1557-9077

Kopp, P.; Muirhead, S.; Jourdain, N.; Gu, W.X.; Jameson, J.L. \& Rodd, C. (1997). Congenital hyperthyroidism caused by a solitary toxic adenoma harboring a novel somatic mutation (serine281-->isoleucine) in the extracellular domain of the thyrotropin receptor. The Journal of Clinical Investigation, Vol.100, No.6, (September 1997), pp. 1634-1639, ISSN 0021-9738

Kratzsch, J. \& Pulzer, F. (2008). Thyroid gland development and defects. Best Practice $\mathcal{E}$ Research: Clinical Endocrinology \& Metabolism, Vol.22, No.1, (February 2008), pp. 5775, ISSN 1521-690X

Lado-Abeal, J.; Castro-Piedras, I.; Palos-Paz, F.; Labarta-Aizpún, J.I. \& Albero-Gamboa, R. (2011). A family with congenital hypothyroidism caused by a combination of lossof-function mutations in the thyrotropin receptor and adenylate cyclasestimulating G alpha-protein subunit genes. Thyroid, Vol.21, No.2, (February 2011), pp. 103-109, ISSN 1557-9077

Libert, F.; Lefort, A.; Gerard, C.; Parmentier, M.; Perret, J.; Ludgate, M.; Dumont, J.E. \& Vassart, G. (1989). Cloning, sequencing and expression of the human thyrotropin 
(TSH) receptor: evidence for binding of autoantibodies. Biochemical and Biophysical Research Communications, Vol.165, No.3, (December 1989), pp. 1250-1255, ISSN 0006$291 X$

Mantovani, G.; Ballare, E.; Giammona, E.; Beck-Peccoz, P. \& Spada, A. (2002). The gsalpha gene: predominant maternal origin of transcription in human thyroid gland and gonads. Journal of Clinical Endocrinology and Metabolism, Vol.87, No.10, (October 2002), pp. 4736-4740, ISSN 0021-972X

Mizuno, H.; Kanda, K.; Sugiyama, Y.; Imamine, H.; Ito, T.; Kato, I.; Togari, H.; Kamoda, T. \& Onigata, K. (2009). Longitudinal evaluation of patients with a homozygous $\mathrm{R} 450 \mathrm{H}$ mutation of the TSH receptor gene. Hormone Research, Vol.71, No.6, (June 2009), pp. 318-323, ISSN 0301-0163

Nagashima, T.; Murakami, M.; Onigata, K.; Morimura, T.; Nagashima, K.; Mori, M. \& Morikawa, A. (2001). Novel inactivating missense mutations in the thyrotropin receptor gene in Japanese children with resistance to thyrotropin. Thyroid, Vol.11, No.6, (June 2001), pp. 551-559, ISSN 1557-9077

Narumi, S.; Muroya, K.; Abe, Y.; Yasui, M.; Asakura, Y.; Adachi, M. \& Hasegawa, T. (2009). TSHR mutations as a cause of congenital hypothyroidism in Japan: a populationbased genetic epidemiology study. Journal of Clinical Endocrinology and Metabolism, Vol.94, No.4, (April 2009), pp. 1317-1323, ISSN 0021-972X

Narumi, S.; Nagasaki, K.; Ishii, T.; Muroya, K.; Asakura, Y.; Adachi, M. \& Hasegawa, T. (2011). Nonclassic TSH resistance: TSHR mutation carriers with discrepantly high thyroidal iodine uptake. Journal of Clinical Endocrinology and Metabolism [Epub ahead of print] (June 2011), ISSN 0021-972X

Nicoletti, A.; Bal, M.; De Marco, G.; Baldazzi, L.; Agretti, P.; Menabò, S.; Ballarini, E.; Cicognani, A.; Tonacchera, M. \& Cassio, A. (2009). Thyrotropin-stimulating hormone receptor gene analysis in pediatric patients with non-autoimmune subclinical hypothyroidism. Journal of Clinical Endocrinology and Metabolism, Vol.94, No.11, (November 2009), pp. 4187-4194, ISSN 0021-972X

Park, S.M. \& Chatterjee, V.K. (2005). Genetics of congenital hypothyroidism. Journal of Medical Genetics, Vol.42, No.5, (May 2005), pp. 379-389, ISSN 0022-2593

Park, S.M.; Clifton-Bligh, R.J.; Betts, P. \& Chatterjee, V.K. (2004). Congenital hypothyroidism and apparent athyreosis with compound heterozygosity or compensated hypothyroidism with probable hemizygosity for inactivating mutations of the TSH receptor. Clinical Endocrinology, Vol.60, No.2, (February 2004), pp. 220-227, ISSN 1365-2265

Refetoff, S. (2003). Resistance to thyrotropin. Journal of Endocrinological Investigation, Vol.26, No.8, (August 2003), pp. 770-779, ISSN 0391-4097

Richter-Unruh, A.; Hauffa, B.P.; Pfarr, N. \& Pohlenz, J. (2004). Congenital primary hypothyroidism in a Turkish family caused by a homozygous nonsense mutation (R609X) in the thyrotropin receptor gene Thyroid, Vol.14, No.11, (November 2004), pp. 971-974, ISSN 1557-9077

Ross, D.S. (2000). Subclinical hypothyroidism. In: Werner and Ingbar's the Thyroid: a Fundamental and Clinical Text, L.E. Braverman, R.D. Utiger, (Eds.), 8th ed., 467-473, 
Lippincott Williams \& Wilkins, ISBN 978-078-1750-47-9, Philadelphia, Pennsylvania, USA

Rubio, I.G.; Galrao, A.L.; Pardo, V.; Knobel, M.; Possato, R.F.; Camargo, R.R.; Ferreira, M.A.; Kanamura, C.T.; Gomes, S.A. \& Medeiros-Neto, G. (2008). A molecular analysis and long-term follow-up of two siblings with severe congenital hypothyroidism carrying the IVS30+1G>T intronic thyroglobulin mutation. Arquivos Brasileiros de Endocrinologia \& Metabologia, Vol.52, No.8, (November 2008), pp. 1337-1344, ISSN 0004-2730

Russo, D.; Betterle, C.; Arturi, F.; Chiefari, E.; Girelli, M.E. \& Filetti, S. (2000). A novel mutation in the thyrotropin (TSH) receptor gene causing loss of TSH binding but constitutive receptor activation in a family with resistance to TSH. Journal of Clinical Endocrinology and Metabolism, Vol.85, No.11, (November 2000), pp. 4238-4242, ISSN 0021-972X

Samuels, M.H.; Schuff, K.G.; Carlson, N.E.; Carello, P.; Janowsky, J.S. (2008). Health status, mood, and cognition in experimentally induced subclinical thyrotoxicosis. Journal of Clinical Endocrinology and Metabolism, Vol.93, No.5, (May 2008), pp. 1730-1736, ISSN 0021-972X

Sriphrapradang, C.; Tenenbaum-Rakover, Y.; Weiss, M.; Barkoff, M.S.; Admoni, O.; Kawthar, D.; Caltabiano, G.; Pardo, L.; Dumitrescu, A.M. \& Refetoff, S. (2011). The coexistence of a novel inactivating mutant thyrotropin receptor allele with two thyroid peroxidase mutations: a genotype-phenotype correlation. Journal of Clinical Endocrinology and Metabolism, Vol.96, No.6, (June 2011), pp. E1001-E1006, ISSN 0021-972X

Stanbury, J.B.; Rocmans, P.; Buhler, U.K. \& Ochi, Y. (1968). Congenital hypothyroidism with impaired thyroid response to thyrotropin. New England Journal of Medicine, Vol.279, No.21, (November 1968), pp. 1132-1136, ISSN 0028-4793

Sunthornthepvarakul, T.; Gottschalk, M.E.; Hayashi, Y. \& Refetoff, S. (1995). Resistance to thyrotropin caused by mutations in the thyrotropin-receptor gene. New England Journal of Medicine, Vol.332, No.3, (January 1995), pp. 155-160, ISSN 0028-4793

Sura-Trueba, S.; Aumas, C.; Carre, A.; Durif, S.; Leger, J.; Polak, M. \& de Roux, N. (2009). An inactivating mutation within the first extracellular loop of the thyrotropin receptor impedes normal posttranslational maturation of the extracellular domain. Endocrinology, Vol.150, No.2, (February 2009), pp. 1043-1050, ISSN 0013-7227

Szkudlinski, M.W.; Fremont, V.; Ronin, C. \& Weintraub, B.D. (2002). Thyroid-stimulating hormone and thyroid-stimulating hormone receptor structure-function relationships. Physiological Reviews, Vol.82, No.2, (April 2002), pp. 473-502, ISSN 0031-9333

Tao, Y.X. (2006). Inactivating mutations of $G$ protein-coupled receptors and diseases: structure-function insights and therapeutic implications. Pharmacology $\mathcal{E}$ Therapeutics, Vol.111, No.3, (September 2006), pp. 949-973, ISSN 0163-7258

Tenenbaum-Rakover, Y.; Grasberger, H.; Mamanasiri, S.; Ringkananont, U.; Montanelli, L.; Barkoff, M.S.; Dahood, A.M. \& Refetoff, S. (2009). Loss-of-function mutations in the thyrotropin receptor gene as a major determinant of hyperthyrotropinemia in a 
consanguineous community. Journal of Clinical Endocrinology and Metabolism, Vol.94, No.5, (May 2009), pp. 1706-1712, ISSN 0021-972X

Tiosano, D.; Pannain, S.; Vassart, G.; Parma, J.; Gershoni-Baruch, R.; Mandel, H.; Lotan, R.; Zaharan, Y.; Pery, M.; Weiss, R.E.; Refetoff, S. \& Hochberg, Z. (1999). The hypothyroidism in an inbred kindred with congenital thyroid hormone and glucocorticoid deficiency is due to a mutation producing a truncated thyrotropin receptor. Thyroid, Vol.9, No.9, (September 1999), pp. 887-894, ISSN 1557-9077

Tonacchera, M.; Van Sande, J.; Cetani, F.; Swillens, S.; Schvartz, C.; Winiszewski, P.; Portmann, L.; Dumont, J.E.; Vassart, G. \& Parma, J. (1996). Functional characteristics of three new germline mutations of the thyrotropin receptor gene causing autosomal dominant toxic thyroid hyperplasia. Journal of Clinical Endocrinology and Metabolism, Vol.81, No.2, (February 1996), pp. 547-554, ISSN 0021$972 X$

Tonacchera, M.; Agretti, P.; Pinchera, A.; Rosellini, V.; Perri, A.; Collecchi, P.; Vitti, P. \& Chiovato, L. (2000). Congenital hypothyroidism with impaired thyroid response to thyrotropin (TSH) and absent circulating thyroglobulin: evidence for a new inactivating mutation of the TSH receptor gene. Journal of Clinical Endocrinology and Metabolism, Vol.85, No.3, (March 2000), pp. 1001-1008, ISSN 0021-972X

Tonacchera, M.; Agretti, P.; De Marco, G.; Perri, A.; Pinchera, A.; Vitti, P. \& Chiovato, L. (2001). Thyroid resistance to TSH complicated by autoimmune thyroiditis. Journal of Clinical Endocrinology and Metabolism, Vol.86, No.9, (September 2001), pp. 45434546, ISSN 0021-972X

Tonacchera, M.; Perri, A.; De Marco, G.; Agretti, P.; Banco, M.E.; Di Cosmo, C.; Grasso, L.; Vitti, P.; Chiovato, L. \& Pinchera, A. (2004). Low prevalence of thyrotropin receptor mutations in a large series of subjects with sporadic and familial nonautoimmune subclinical hypothyroidism. Journal of Clinical Endocrinology and Metabolism, Vol.89, No.11, (November 2004), pp. 5787-5793, ISSN 0021-972X

Tonacchera, M.; Di Cosmo, C.; De Marco, G.; Agretti, P.; Banco, M.; Perri, A.; Gianetti, E.; Montanelli, L.; Vitti, P. \& Pinchera, A. (2007). Identification of TSH receptor mutations in three families with resistance to TSH. Clinical Endocrinology, Vol.67, No.5, (November 2007), pp. 712-718, ISSN 1365-2265

Tsunekawa, K.; Onigata, K.; Morimura, T.; Kasahara, T.; Nishiyama, S.; Kamoda, T.; Mori, M.; Morikawa, A. \& Murakami, M. (2006). Identification and functional analysis of novel inactivating thyrotropin receptor mutations in patients with thyrotropin resistance. Thyroid, Vol.16, No.5, (May 2006), pp. 471-479, ISSN 1557-9077

Utiger, R.D. (1995). Thyrotropin-receptor mutations and thyroid dysfunction. New England Journal of Medicine, Vol.332, No.4, (January 1995), pp. 183-185, ISSN 0028-4793

Van Durme, J.; Horn, F.; Costagliola, S.; Vriend, G. \& Vassart, G. (2006). GRIS: glycoproteinhormone receptor information system. Molecular Endocrinology, Vol.20, No.9, (September 2006), pp. 2247-2255, ISSN 0888-8809

Van Sande, J.; Parma, J.; Tonacchera, M.; Swillens, S.; Dumont, J. \& Vassart, G. (1995). Somatic and germline mutations of the TSH receptor gene in thyroid diseases. Journal of Clinical Endocrinology and Metabolism, Vol.80, No.9, (September 1995), pp. 2577-2585, ISSN 0021-972X 
Vassart, G. \& Dumont, J.E. (1992). The thyrotropin receptor and the regulation of thyrocyte function and growth. Endocrine Reviews, Vol.13, No.3, (August 1992), pp. 596-611, ISSN 0163-769X

Wonerow, P.; Neumann, S.; Gudermann, T. \& Paschke, R. (2001). Thyrotropin receptor mutations as a tool to understand thyrotropin receptor action. Journal of Molecular Medicine, Vol.79, No.12, (December 2001), pp. 707-721, ISSN 0946-2716

Xie, J.; Pannain, S.; Pohlenz, J.; Weiss, R.E.; Moltz, K.; Morlot, M.; Asteria, C.; Persani, L.; Beck-Peccoz, P.; Parma, J.; Vassart, G. \& Refetoff, S. (1997). Resistance to thyrotropin (TSH) in three families is not associated with mutations in the TSH receptor or TSH. Journal of Clinical Endocrinology and Metabolism, Vol.82, No.12, (December 1997), pp. 3933-3940, ISSN 0021-972X 


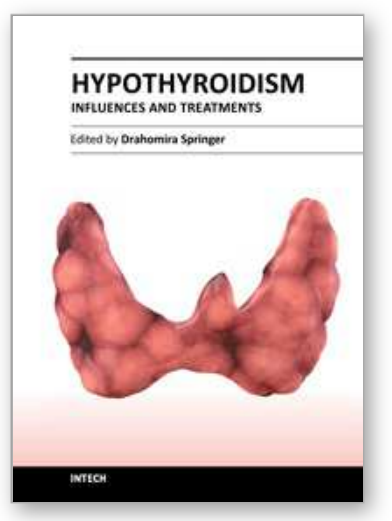

\author{
Hypothyroidism - Influences and Treatments \\ Edited by Dr. Drahomira Springer
}

ISBN 978-953-51-0021-8

Hard cover, 348 pages

Publisher InTech

Published online 08, February, 2012

Published in print edition February, 2012

Hypothyroidism is the most common thyroid disorder and it is significantly more frequent than presented millions of people suffer from this disease without knowing it. People with this condition will have symptoms associated with slow metabolism. Estimates of subclinical hypothyroidism range between 3 to $8 \%$, increasing with age, whereas it more likely affects women than men. About $10 \%$ of women may have some degree of thyroid hormone deficiency. Hypothyroidism may affect lipid metabolism, neurological diseases or other clinical conditions. The book includes studies on advancements in diagnosis, regulation and replacement therapy, thyroid ultrasonography and radioiodine therapy for hypothyroidism. "Hypothyroidism - Influences and Treatments" contains many important specifications, results of scientific studies and innovations for endocrine practice.

\title{
How to reference
}

In order to correctly reference this scholarly work, feel free to copy and paste the following:

Yardena Tenenbaum-Rakover (2012). The Clinical Spectrum of Thyrotropin Receptor Gene (TSHR)

Mutations, Hypothyroidism - Influences and Treatments, Dr. Drahomira Springer (Ed.), ISBN: 978-953-510021-8, InTech, Available from: http://www.intechopen.com/books/hypothyroidism-influences-andtreatments/the-clinical-spectrum-of-tsh-receptor-tshr-mutations

\section{INTECH}

open science | open minds

\author{
InTech Europe \\ University Campus STeP Ri \\ Slavka Krautzeka 83/A \\ 51000 Rijeka, Croatia \\ Phone: +385 (51) 770447 \\ Fax: +385 (51) 686166 \\ www.intechopen.com
}

\author{
InTech China \\ Unit 405, Office Block, Hotel Equatorial Shanghai \\ No.65, Yan An Road (West), Shanghai, 200040, China \\ 中国上海市延安西路65号上海国际贵都大饭店办公楼 405 单元 \\ Phone: +86-21-62489820 \\ Fax: +86-21-62489821
}


(C) 2012 The Author(s). Licensee IntechOpen. This is an open access article distributed under the terms of the Creative Commons Attribution 3.0 License, which permits unrestricted use, distribution, and reproduction in any medium, provided the original work is properly cited. 